\title{
La revalidation des médecins au Canada : Passons-nous le test?
}

$\mathrm{L}$ es médecins sont tenus par l'éthique de maintenir leur compétence dans leur discipline, comme élément de leur obligation professionnelle de rendre compte au public. Dans son Code de déontologie, l'Association médicale canadienne affirme que les médecins doivent «chercher à approfondir toujours leur savoir afin de préserver et d'améliorer leurs connaissances, compétences

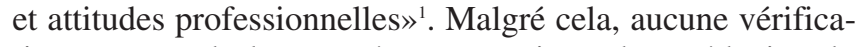
tion externe de la compétence continue des médecins du Canada n'est exigée et la profession ne peut donc prouver au public qu'elle applique les normes les plus rigoureuses.

À l'heure actuelle, les processus qui garantissent que les médecins maintiennent leur compétence dépendent des décisions réglementaires indépendantes et hétérogènes prises par le Collège des médecins et chirurgiens de chaque province. Trois provinces (Saskatchewan, Ontario et Québec) exigent actuellement que les médecins participent à un programme de formation visant à conserver leur permis d'exercice. Les médecins satisfont habituellement à cette exigence en participant au Programme de maintien du certificat du Collège royal des médecins et chirurgiens ou au Programme de maintien de la compétence du Collège des médecins de famille. Ces programmes visent les bons éléments conceptuels de maintien de la compétence, mais comme ils sont de nature autodéclaratoire, ils n'ont pas la rigueur voulue pour obliger les médecins à rendre compte. Une autre stratégie, appliquée en Alberta et en Nouvelle-Écosse, oblige les médecins à participer à un mécanisme d'examen au cours duquel on leur présente des commentaires de patients et de pairs au sujet de leur travail. Le programme est conçu pour faciliter l'amélioration de la qualité, mais ne comporte pas de norme externe de «réussite». De plus, la méthode n'est applicable que pour les soins primaires et les spécialités de plus grande envergure, parce que l'examen par les pairs est difficile dans les spécialités qui comptent peu de membres.

Les voix du changement exigent maintenant un mécanisme plus transparent et redditionnel de maintien des normes professionnelles. En 2007, la Fédération des ordres des médecins du Canada affirmait dans un énoncé de principe que «tous les médecins autorisés du Canada doivent participer à un mécanisme de revalidation reconnu dans lequel ils et elles démontrent leur engagement envers le maintien d'une performance compétente dans un cadre d'équité, de pertinence, d'inclusivité, de transférabilité et de formation ${ }^{2} . »$ On y affirmait que les programmes administrés par le Collège royal des médecins et chirurgiens et le Collège des médecins de famille établissent un cadre normatif canadien de développement professionnel, mais qu'ils ne peuvent toutefois prouver que les médecins utilisent vraiment leurs connaissances et leurs compétences. Même si la Fédération des ordres des médecins du Canada n'a pas le pouvoir d'imposer des changements, l'appui général que l'énoncé de position a reçu des organisations médicales nationales pousse les ordres des provinces à envisager des changements de politique. Des exemples très médiatisés d'infractions graves aux normes de pratique de la médecine par certains médecins, comme les cas récents d'erreurs en pathologie au Nouveau-Brunswick et à Terre-Neuve, préoccupent avec raison les médias et le public, qui se demandent si l'examen actuel des médecins par leurs pairs est suffisamment rigoureux ou si une réglementation de l'extérieur s'impose. La barre monte encore ailleurs, mais le Canada ne suit pas : d'autres pays comme le Royaume-Uni et les ÉtatsUnis ont déjà mis en œuvre des mécanismes de revalidation plus exigeants que ceux qu'imposent actuellement la plupart des provinces canadiennes.

Comme spécialiste active en médecine interne au Canada, j'ai participé au Programme de maintien du certificat du Collège royal. Chaque année, je présente mes crédits de participation à des activités d'éducation médicale continue au cours desquelles j'assiste à des conférences, je réponds à des questions cliniques pertinentes à ma pratique et je participe à un programme d'autoévaluation. Personne ne me soumet à des examens pour déterminer ce que j'ai appris ou si j'ai appliqué ces connaissances. Par contre, j'ai participé récemment au processus de maintien du certificat de l'American Board of Internal Medicine, que j'ai trouvé beaucoup plus difficile et exigeant. Depuis 1990, tous les spécialistes aux États-Unis doivent renouveler leur certificat aux 10 ans pour conserver le titre de spécialiste certifié. Le processus oblige à participer à deux éléments qui n'existent pas au Canada : un exercice d'amélioration de la pratique et un examen protégé. Le module d'amélioration de la pratique consiste notamment à recueillir des données sur le rendement actuel au travail dans un domaine précis (p. ex., prise en charge du diabète), à évaluer l'organisation du bureau qui appuie les soins et à sonder les patients pour connaître leur expérience. Ces données sont soumises à l'American Board of Internal Medicine et les médecins reçoivent des commentaires où l'on établit une comparaison avec les normes établies et avec leurs pairs. Les médecins doivent aussi présenter un plan d'amélioration de la qualité. L'examen protégé est un test informatisé à choix multiples portant sur les techniques de cognition dans le domaine de pratique. Pour me préparer au volet examen protégé, j’ai utilisé un programme d'autoévaluation des connaissances afin de réviser l'ensemble de la médecine interne générale. L'amélioration de la pratique et la préparation à l'examen ont toutes deux exigé beaucoup plus de temps que le processus du Collège royal. J'ai étudié pendant quatre mois et j'ai eu peur d'échouer. Je n'aurais jamais fait cet effort si je n'avais pas eu à me présenter à un examen.

Devrions-nous tous avoir à passer un examen à intervalles réguliers? Les données montrent que le public appuie le concept de l'examen périodique du savoir des médecins. Selon un sondage, $87 \%$ des patients croient que cela devrait se 
faire ${ }^{3}$. De plus, des données démontrent que les résultats obtenus à un examen peuvent être un indicateur de rendement. Au Québec, les chercheurs ont constaté qu'il y avait un lien entre le résultat obtenu par les médecins de famille à leur examen de certification et à l'examen d'aptitude du Conseil médical du Canada et la prestation de soins de qualité après quatre à sept ans de pratique 4 . Plus récemment, Holmboe et ses collaborateurs ont constaté qu'il y avait un lien entre les résultats obtenus par les médecins à l'examen de maintien du certificat de l'American Board of Internal Medicine et des taux plus élevés de rendement dans le soin des patients couverts par le régime Medicare ${ }^{5}$. Même si les connaissances que vérifie l'examen ne constituent pas la seule mesure de la compétence, il s'agit d'une dimension importante d'un diagnostic exact et de la prise de décision clinique. Il faut sans aucun doute pousser les recherches plus loin pour comprendre le lien entre la revalidation et la qualité des soins dans la pratique et explorer les façons les plus efficaces d'évaluer le maintien des connaissances, des compétences et des attitudes des médecins.

Les médecins du Canada et les ordres des provinces devraient implanter la revalidation de tous les médecins. Les normes doivent inclure une évaluation externe de la compétence au lieu de compter exclusivement sur l'autoévaluation. Il faut un examen externe rigoureux afin d'obliger les médecins à faire un effort pour réussir. Même s'il faut plus de données pour déterminer le meilleur mécanisme de revalidation, le processus devrait inclure une évaluation externe à la fois du savoir médical et de l'aptitude réelle du médecin à dispenser des soins de qualité dans sa pratique. Un examen protégé comme celui des États-Unis pourrait évaluer le savoir et un examen de la pratique comportant l'apport de pairs et de patients pourrait permettre d'évaluer le rendement réel. Des normes nationales devraient déterminer les exigences minimales.

Les répercussions du resserrement des exigences préoccupent inévitablement les médecins : crainte de l'échec, obligation de se préparer et effet possible d'une perte des médecins qui ne réussissent pas. Ces préoccupations seraient probablement transitoires. Aux États-Unis, les programmes de renouvellement du certificat se sont butés au début à la résistance des médecins, mais on s'attend maintenant à ce que tous les nouveaux diplômés se soumettent à la revalidation. En bout de ligne, nous devons pouvoir garantir à nos collègues et au public que nous sommes à la hauteur de la responsabilité professionnelle que nous impose l'autoréglementation et que nous protégeons la confiance du public dans notre système de santé.

\section{Wendy Levinson MD}

Professeure et titulaire de la Chaire Sir John and Lady Eaton Département de médecine

Université de Toronto

Toronto (Ont.)

Avec l'équipe de rédaction de l'éditorial

(Paul C. Hébert MD MHSc, Rajendra Kale MD,

Matthew B. Stanbrook MD PhD, Barbara Sibbald BJ, Ken Flegel MDCM MSc et Amir Attaran LLB DPhil)

Intérêts concurrents: Wendy Levinson est la présidente désignée de l'American Board of Internal Medicine. Voir www.cmaj.ca/misc/edboard.shtml pour les déclarations de l'équipe de rédaction de l'éditorial.

Traduit par le Service de traduction de l'AMC.

\section{RÉFÉRENCES}

1. Association médicale canadienne. Code de déontologie de l'AMC. Ottawa (Ont.) : L'Association; 2004. Disponible à : http://policybase.cma.ca/dbtw-wpd/PolicyPDF /PD04-06F.pdf (consulté le 25 août 2008).

2. Groupe de travail sur la revalidation de la Fédération des ordres des médecins du Canada. La revalidation des médecins : Maintien des compétences et de la performance. Ottawa (Ont.) : Fédération des ordres des médecins du Canada; 2007. Disponible : www.fmrac.ca/committees/documents/final_reval_position_french .pdf (consulté le 9 septembre 2008).

3. Brennan TA, Horwitz RI, Duffy FD, et al. The role of physician specialty board certification status in the quality movement. JAMA 2004;292:1038-43.

4. Tamblyn R, Abrahamowicz M, Dauphinee WD, et al. Association between licensure examination scores and practice in primary care. JAMA 2002;288:3019-26.

5. Holmboe ES, Wang Y, Meehan T, et al. Association between maintenance of certification examination scores and quality of care for medicare beneficiaries. Arch Intern Med 2008;168:1396-403. 